\title{
Questioning dogma: does a GCS of 8 require intubation?
}

\author{
Justin S. Hatchimonji ${ }^{1}$ (I) Ryan P. Dumas ${ }^{2}$ - Elinore J. Kaufman ${ }^{3} \cdot$ Dane Scantling $^{3} \cdot$ Jordan B. Stoecker $^{1}$. \\ Daniel N. Holena ${ }^{3,4}$
}

Received: 18 March 2020 / Accepted: 24 April 2020 / Published online: 7 May 2020

(c) Springer-Verlag GmbH Germany, part of Springer Nature 2020

\begin{abstract}
Background There is no evidence supporting intubation for a Glasgow Coma Scale (GCS) of 8. We investigated the effect of intubation in trauma patients with a GCS 6-8, with the hypothesis that intubation would increase mortality and length of stay. Methods We studied adult patients with GCS 6-8 from the 2016 National Trauma Data Bank. Intubated and non-intubated patients were compared using inverse probability weighted regression adjustment (IPWRA) to control for injury severity and patient characteristics. Outcomes were mortality, intensive care unit length of stay (ICU LOS), and total LOS. Stratified analysis was performed to investigate the effect in patients with and without head injuries.

Results Among 6676 patients with a GCS between 6 and 84,078 were intubated within $1 \mathrm{~h}$ of arrival to the emergency department. The overall mortality rate was $15.1 \%$. IPWRA revealed an increase in mortality associated with intubation (OR $1.05,95 \%$ CI 1.03, 1.06). The results were similar in patients with head injuries (OR 1.04, 95\% CI 1.02, 1.06) and without (OR 1.06, 95\% CI 1.03, 1.10). Among the 5,742 patients admitted to the ICU, intubation was associated with a $14 \%$ increase in ICU LOS (95\% CI 8-20\%; 5.5 vs. 4.8 days; $p<0.001)$. The overall length of stay was $27 \%$ longer (95\% CI $19.8-34.3 \%)$ among intubated patients (mean 7.7 vs 6.0 days; $p<0.001$ ).

Conclusion Among patients with GCS of 6 to 8, intubation on arrival was associated with an increase in mortality and with longer ICU and overall length of stay. The use of a strict threshold GCS to mandate intubation should be revisited.
\end{abstract}

Keywords Trauma $\cdot$ Intubation $\cdot$ Quality improvement $\cdot$ Processes of care

\section{Introduction}

Endotracheal intubation for oxygenation, ventilation, and airway protection is a key intervention in trauma care, but it is not without risks. The American College of Surgeons

The material has been presented at Academic Surgical Congress 2020, Orlando, FL.

Justin S. Hatchimonji

justin.hatchimonji@pennmedicine.upenn.edu

Ryan P. Dumas

ryan.dumas@UTSouthwestern.edu

Elinore J. Kaufman

elinore.kaufman@pennmedicine.upenn.edu

Dane Scantling

dane.scantling@pennmedicine.upenn.edu

Jordan B. Stoecker

jordan.stoecker@pennmedicine.upenn.edu

Daniel N. Holena

daniel.holena@uphs.upenn.edu
Committee on Trauma Advanced Trauma Life Advanced Trauma Life Support (ATLS) course teaches that a Glasgow Coma Scale (GCS) score of 8 or lower mandates intubation for airway protection [1]. Likewise, the Eastern Association for the Surgery of Trauma (EAST) practice management guidelines (PMGs) give a level 1 recommendation for intubation for patients with a GCS of $8[2,3]$. Indeed, real-world

1 Department of Surgery, Perelman School of Medicine at the University of Pennsylvania, 3400 Spruce St, 4 Maloney, Philadelphia, PA, USA

2 Division of General and Acute Care Surgery, Department of Surgery, University of Texas Southwestern MedicalCenter at Dallas, Dallas, TX, USA

3 Division of Traumatology, Surgical Critical Care and Emergency Surgery, Perelman School of Medicine at the University of Pennsylvania, Philadelphia, PA, USA

4 Center for Clinical Epidemiology and Biostatistics, Perelman School of Medicine at the University of Pennsylvania, Philadelphia, PA, USA 
trauma center practice varies, and some clinicians may have an even more conservative threshold for intubation. A review of one center's indications for intubation revealed that $25 \%$ of patients were intubated for "altered mental status", but had a GCS $>8$ [4]. Following this, a 2012 update to the aforementioned EAST guidelines gave a level 3 recommendation for intubating patients with "moderate" cognitive impairment (GCS 9-12) [3].

Despite these high-level recommendations, there is no direct evidence to support this practice. Endotracheal intubation preserves oxygenation and ventilation and may prevent aspiration in injured patients, but intubation also puts patients at risk of hypotension, exposes them to sedating and paralyzing medications, risks ventilator-associated complications, and usually mandates an intensive care unit stay. Therefore, while the "GCS 8" rule has reasonable face validity, it deserves more careful consideration.

Given the lack of strong evidentiary support for intubating patients for a GCS of 8 or less, we sought to investigate the treatment effect of intubation in trauma patients with a GCS less than 8 using a nationally representative database. We recognize that obtunded patients with a severely depressed GCS are likely to benefit from intubation, but suggest that there is an inherent difference between those who fall in the bottom quartile of GCS and those in the range of 6-8. Indeed, there is some support for this in the literature $[5,6]$. Therefore, we chose to study patients with a "marginal" GCS, under the hypothesis that for patients with a GCS between 6 and 8, intubation would have no effect on mortality, but would increase the length of stay.

\section{Patients and methods}

We conducted a retrospective cohort study using the 2016 National Trauma Data Bank (NTDB) [7]. The NTDB is a centralized, nationally representative dataset created and maintained by the American College of Surgeons (ACS). Over 700 centers contribute to it across all levels of trauma designation; contribution to the NTDB is an ACS requirement for Level I and II centers. This dataset is frequently used in large-scale retrospective studies [8].

We excluded patients that were transferred to another hospital, either from the emergency department or later in their hospital stay because we could not ascertain the primary outcomes of interest in these patients. We excluded patients aged $<16$ years, patients who arrived without signs of life or vital signs indicating they were dead on arrival, and those with an advance directive limiting care.

Intubation was identified using procedure codes. We counted patients as having been intubated on arrival if they carried an ICD-9 or ICD-10 code for endotracheal intubation and the timestamp associated with that code was within $1 \mathrm{~h}$ of arrival. A summary of included ICD codes is shown in Table 5 in Appendix 1. We also defined patients as 'intubated' if their ED dispositions were listed as "operating room," indicating that they underwent immediate operation and thus required intubation. These patients accounted for a minority (10.4\%) of the intubated cohort. A patient was considered to have been intubated in the field if there was no intubation code, but his or her initial GCS carried an NTDB qualifier code indicating that they were intubated, sedated, or chemically paralyzed. These patients were excluded. A flow diagram of inclusions and exclusions is shown in Fig. 1.

Hypotension was defined as a presenting systolic blood pressure (SBP) of less than $90 \mathrm{mmHg}$. The data were inspected for missingness. Of the 6676 patients, 4 were missing a value for mortality, 2 for sex, 333 for race, and 238 for hospital length of stay. Aside from intensive care unit (ICU) length of stay (LOS), 92\% of patients in this cohort had complete records. To reduce the risk of bias associated with casewise deletion, we used multiple imputation with chained equations [9]. With respect to ICU LOS, the majority of those missing values $(926 / 934 ; 99.1 \%)$ were missing because the patients were noted not to have been admitted to the ICU. Thus, the analysis treating ICU LOS as the outcome was performed as a complete-case analysis.

We next tabulated descriptive statistics between those that were intubated and those that were not. Differences were expressed using Pearson's chi-squared, Student's $t$ test, or Mann-Whitney $U$ test, as appropriate. The primary exposure variable in our analysis was ED intubation. The primary outcome was mortality. Secondary outcomes included ICU LOS and overall hospital LOS. To explore potential bias incurred by the inclusion of direct-to-operating room patients that were not otherwise coded as intubated, we conducted a sensitivity analysis in which the mortality risk was re-examined, excluding these patients. In a sub-analysis of mortality risk, the cohort was stratified by head injury, as defined as any abbreviated injury scale (AIS) head score $>0$. A sensitivity analysis was also performed for the stratification; for this analysis, we redefined head injury as AIS $>1$. Finally, as intoxication may be a factor that influences clinicians' decisions to observe patients without intubation, we performed a separate analysis, stratified by alcohol intoxication. For these purposes, intoxication was defined as a measured level above the legal limit. To control for potential confounders, regression analyses (logistic for the primary outcome and linear for the secondary outcomes) were used. Because both LOS variables had skewed distributions, we log-transformed them before using them as outcomes in our linear regression models. Furthermore, given that the likelihood of exposure (intubation) is dependent on several factors likely to be correlated with the outcome (mortality) - including GCSwe used inverse probability weighting to adjust for severity of illness and patient factors. 


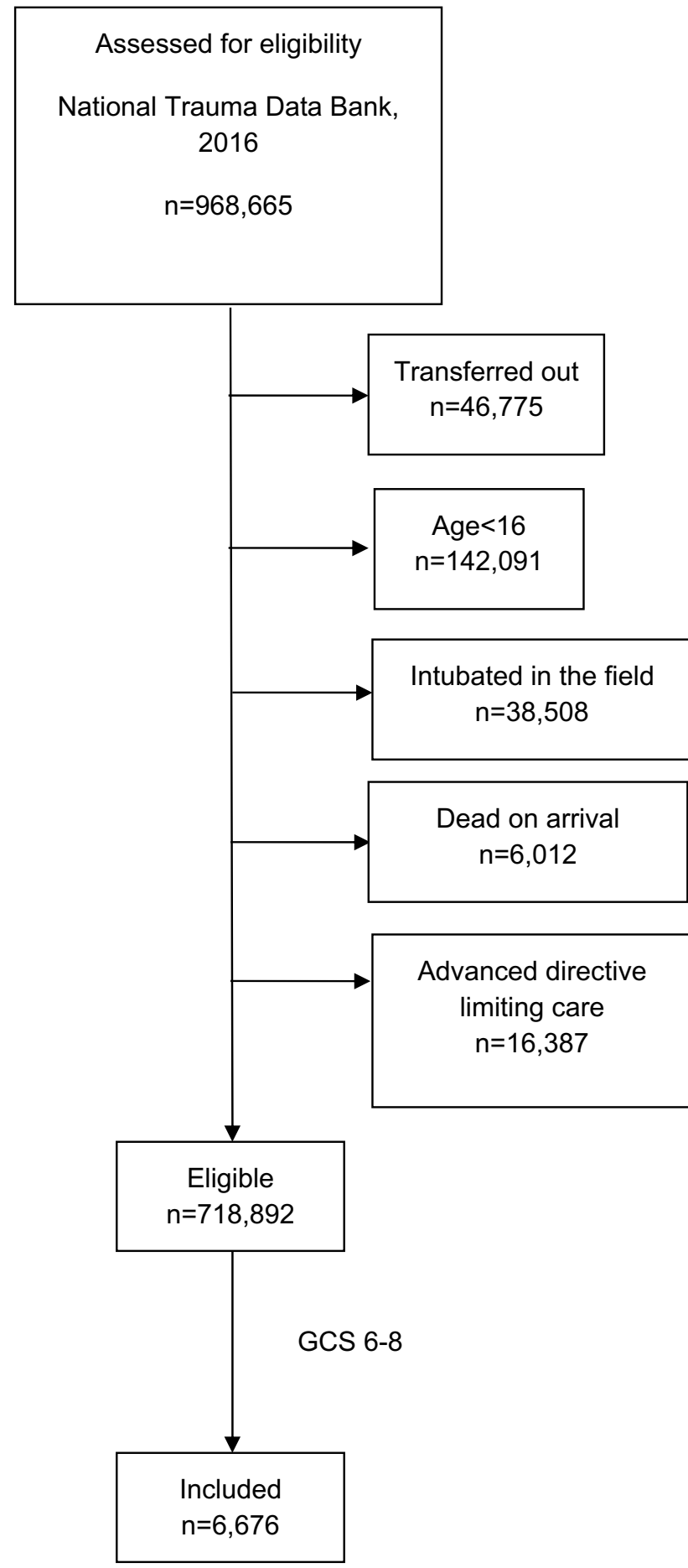

Fig. 1 Inclusions and exclusions

Inverse probability weighting (IPW) is one method of reducing confounding in observational studies. For example, severity of injury, deranged physiology, and GCS are known to be associated with the outcome of mortality but may also be associated with intubation, the exposure of interest. To mitigate this confounding, IPW uses propensity scores (PS), [10] which employ a regression analysis to estimate a patient's likelihood of exposure, to 'weight' each patient such that those that are less likely to be exposed contribute more 'weight' than those who are more likely [11]. PS and IPW can be used in a variety of ways; in inverse probability weight regression adjustment (IPWRA), a regression model is used to estimate PS (and therefore IPW =1/PS); another regression model including several covariates as well as the PSs is fitted to estimate the strength of an association with the outcome of interest [12]. This is often expressed as an average treatment effect (ATE), which is the theoretical average of the differences between treatment and exposure outcomes for each patient. This treatment effect is unobservable at the individual level as we cannot know the outcome given the counterfactual for any given patient, but these statistical methods allow us to estimate the average effect [13].

This study was determined by our Institutional Review Board to be exempt from review. The data contained here were obtained from the National Trauma Data Bank (NTDB). The content reproduced from the NTDB remains the full and exclusive property of the American College of Surgeons. The American College of Surgeons is not responsible for any claims arising from works based on the original data, text, tables, or figures. All statistical analyses were performed using Stata version 15.1 (College Station, TX). A two-tailed $p$ value of $<0.05$ was considered significant in the final analysis.

\section{Results}

After exclusions, there were 718,892 patients remaining; 6676 had a GCS score of 6-8 and were included in the analysis. 4,078 were intubated within $1 \mathrm{~h}$ of arrival to the ED. Table 1 shows patient characteristics. Intubated patients had a higher mortality rate (17.5\% vs $11.4 \%)$. There was an association between lower GCS score and intubation ( $64.3 \%$ of patients with GCS $=6$ intubated vs $56.9 \%$ with $\mathrm{GCS}=8$ ). Intubated patients were also younger, more often males, more often hypotensive on arrival ( $8.7 \%$ vs $4.5 \%$ ), and more severely injured (median ISS 19 vs 10).

Using IPWRA analysis adjusted for GCS, age, sex, hypotension, maximum head, chest, and abdomen/pelvis abbreviated injury scale (AIS) scores, we determined that the average treatment effect (ATE) of intubation was associated with increased odds of mortality (OR 1.05, 95\% CI 1.03-1.06). In both the intubated and non-intubated groups, increasing severity of injury, hypotension, age, and decreasing GCS were also associated with increased mortality. The results are summarized in Table 2 . In a sensitivity analysis excluding the 424 patients that were not otherwise coded for intubation but proceeded from trauma bay to operating room, the associated mortality risk was the same (OR 1.05, 95\% CI 1.03-1.06).

There were 1,524 head-injured patients (defined by any head AIS >0). In our stratified analysis, a similar result was 
Table 1 Patient characteristics

\begin{tabular}{llll}
\hline & Not intubated $(n=2598)$ & Intubated $(n=4078)$ & $p$ value \\
\hline $\begin{array}{l}\text { Died, (\%) } \\
\text { Total GCS score }\end{array}$ & $715(17.5 \%)$ & $<0.001$ \\
6 & $719(35.7 \%)$ & $1293(64.3 \%)$ & $<0.001$ \\
7 & $866(32.5 \%)$ & $1446(62.5 \%)$ & \\
8 & $1013(43.1 \%)$ & $1339(56.9 \%)$ & $<0.001$ \\
Age, years & $47.3 \pm 21.1$ & $43.0 \pm 19.5$ & $<0.001$ \\
Female (\%) & $727(28.0 \%)$ & $977(24.0 \%)$ & $<0.001$ \\
Hypotensive on arrival (\%) & $117(4.5 \%)$ & $353(8.7 \%)$ & $<0.001$ \\
ISS & $10(5,22)$ & $19(10,27)$ & \\
\hline
\end{tabular}

ISS expressed as median (interquartile range $=\mathrm{IQR}$ ) and analyzed using Mann-Whitney $U$ test

Age expressed as mean \pm SD and analyzed using two-sample $t$ Test. GCS, sex, mortality, and hypotension analyzed using Pearson's chi-squared test. Column percentages are presented, except for GCS, which is presented in row percentages. Hypotension defined as systolic blood pressure $<90 \mathrm{mmHg}$ on arrival

GCS Glasgow Coma Scale, ISS injury severity score

Table 2 Results of inverse probability weighted with regression adjustment (IPWRA) logistic regression analysis (outcome: in-hospital mortality)

\begin{tabular}{|c|c|c|c|c|c|c|}
\hline \multirow[t]{2}{*}{ Factor } & \multicolumn{3}{|c|}{ Intubated $(n=4078)$} & \multicolumn{3}{|c|}{ Not intubated $(n=2598)$} \\
\hline & OR & $95 \% \mathrm{CI}$ & $p$ value & OR & $95 \% \mathrm{CI}$ & $p$ value \\
\hline Intubation [ATE] & 1.05 & $1.03-1.06$ & $<0.001$ & & & \\
\hline Total GCS (per point) & 0.71 & $0.63-0.80$ & $<0.001$ & 0.76 & $0.63-0.93$ & 0.008 \\
\hline Age (per year) & 1.05 & $1.04-1.05$ & $<0.001$ & 1.06 & $1.05-1.08$ & $<0.001$ \\
\hline Female & 0.88 & $0.71-1.09$ & 0.23 & 0.59 & $0.42-0.84$ & 0.003 \\
\hline Hypotensive & 1.95 & $1.40-2.72$ & $<0.001$ & 4.33 & $2.33-8.05$ & $<0.001$ \\
\hline Maximum head AIS (per point) & 1.54 & $1.44-1.64$ & $<0.001$ & 1.95 & $1.71-2.21$ & $<0.001$ \\
\hline Maximum chest AIS (per point) & 1.12 & $1.04-1.20$ & 0.002 & 1.24 & $1.07-1.42$ & 0.003 \\
\hline Maximum Abdominal AIS (per point) & 1.36 & $1.23-1.49$ & $<0.001$ & 0.98 & $0.78-1.23$ & 0.87 \\
\hline
\end{tabular}

OR for intubation shown as average treatment effect (ATE). ORs for covariates shown in the treated and untreated (intubated and not intubated, respectively)

OR odds ratio, $C I$ confidence interval, GCS Glasgow Coma Scale, AIS abbreviated injury scale
Table 3 Odds of mortality with intubation, stratified by head injury

\begin{tabular}{llll}
\hline & $\begin{array}{l}\text { OR, mortality with } \\
\text { intubation (ATE) }\end{array}$ & $95 \%$ CI & $p$ value \\
\hline $\begin{array}{c}\text { Head injured } \\
(n=5152)\end{array}$ & 1.04 & $1.02-1.05$ & $<0.001$ \\
$\begin{array}{c}\text { Non-head injured } \\
(n=1524)\end{array}$ & 1.06 & $1.03-1.10$ & $<0.001$ \\
\hline
\end{tabular}

Determined by inverse probability weighted logistic regression with regression adjustment (IPWRA)

$O R$ odds ratio, ATE average treatment effect, $C I$ confidence interval

seen in the head injured (OR 1.04, 95\% CI 1.02-1.05) and non-head injured (OR 1.06, 95\% CI 1.03-1.10) (Table 3). When the definition of head injured was adjusted to AIS > 1, similar results were again observed: OR $1.03(95 \%$ CI 1.01-1.06) in the head injured and OR 1.05 (95\% CI $1.03-1.08)$ in the non-head injured.

$1,991 / 6,676$ patients $(29.8 \%)$ were identified as intoxicated on arrival. $3,132(46.9 \%)$ were tested and found not to be intoxicated, leaving $23.3 \%$ of the cohort with missing data on intoxication. In a stratified analysis, intoxicated patients carried a mortality OR of 1.03 (95\% CI 1.01-1.05) with intubation, while non-intoxicated patients carried a mortality OR of 1.05 (95\% CI 1.03-1.08).

Intubation was associated with a statistically significant $13.7 \%$ increase in ICU LOS (95\% CI 8.0-19.6\%) and a $26.8 \%$ increase in overall length of stay $(95 \% \mathrm{CI}$ $19.8-34.3 \%$ ), translating to mean increases from 4.8 to 5.5 days and 6.0 to 7.7 days, respectively (Table 4) (Fig. 2).

\section{Discussion}

For patients with a GCS between 6 and 8, we found that intubation was associated with increased odds of mortality. This association was consistent irrespective of the presence of head injury. Furthermore, intubation was associated with longer ICU and hospital length of stay. These findings bring 
Table 4 Increase in intensive care unit (ICU) and overall length of stay (LOS) with intubation

\begin{tabular}{llllll}
\hline & $\begin{array}{l}\text { \% increase in days } \\
\text { with intubation } \\
\text { (ATE) }\end{array}$ & $95 \%$ CI & $\begin{array}{l}\text { Mean \# days } \\
\text { without intuba- } \\
\text { tion }\end{array}$ & $\begin{array}{l}\text { Mean \# days } \\
\text { with intubation }\end{array}$ & $p$ value \\
\hline ICU LOS $(n=5742)$ & 13.7 & $8.0-19.6$ & 4.8 & 5.5 & $<0.001$ \\
Overall LOS $(n=6676)$ & 26.8 & $19.8-34.3$ & 6.0 & 7.7 & $<0.001$ \\
\hline
\end{tabular}

Determined by inverse probability weighted linear regression with regression adjustment (IPWRA) on logtransformed data. The analysis of overall LOS performed using multiply imputed values. ICU LOS analysis performed excluding patients missing a value for ICU LOS

$A T E$ average treatment effect, $C I$ confidence interval

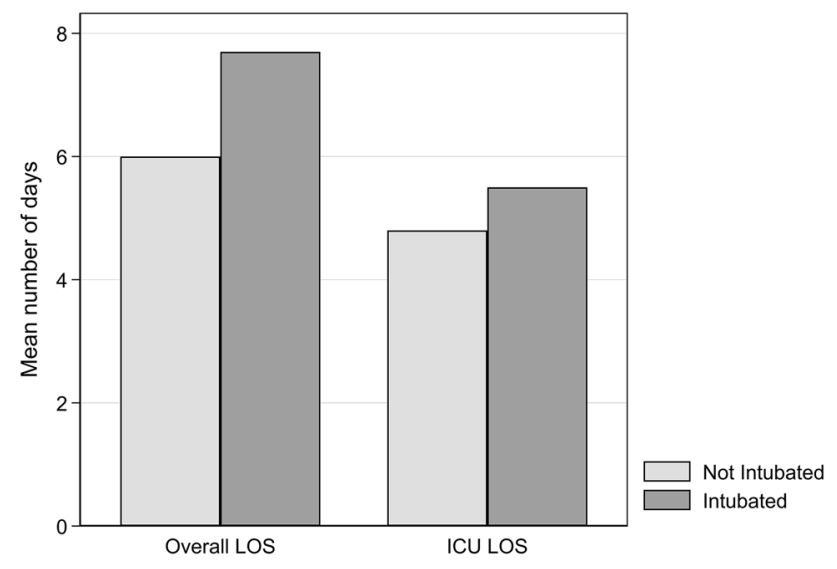

Fig. 2 Mean ICU and overall LOS, by intubation status. ICU, intensive care unit; LOS, length of stay

into question the established dogma that mandates intubation for GCS $\leq 8$.

As stated in the Introduction, EAST gives a level 1 recommendation to intubate this cohort of patients $[2,3]$. However, many of the studies cited in the PMG were designed to answer different questions or study different subsets of these patients, including the safety of cricothyroidotomy [14] or neuromuscular blockade, [15] and a comparison of early vs late intubation [16]. Of the 31 studies cited as supporting evidence for this recommendation, 16 were for the purposes of studying prehospital intubation. This is not to say that these studies (or the guidelines based on them) are not valuable, but they do not constitute direct evidence that there is an outcome benefit to intubating all trauma patients presenting with GCS $\leq 8$.

However, there is some existing evidence for the potential harms of intubation in trauma patients. One study examining the effect of intubating patients for "combativeness" found that these patients, as compared to a matched non-intubated group, incurred longer hospital length of stay, increased rates of pneumonia, and poorer discharge status [17]. Furthermore, there is some literature to suggest that it may be appropriate to observe some patients with a GCS less than 8 without intubation. A small observational study, noting that many emergency department providers advocate intubation for GCS $\leq 8$ in intoxicated (non-trauma) patients, reported safe management without intubation in all of the included GCS $\leq 8$ patients [18]. There may be a similar cohort of trauma patients who, despite impaired cognition, may be safely managed without intubation. However, this is the first study to our knowledge of the effect of intubation on trauma patients with a marginal GCS.

As a retrospective cohort study with a small effect size, this work could be categorized as Level III evidence [19]. However, this modest increase in mortality risk is less impactful for practice than the knowledge that there is no decrease in mortality risk. This, after all, is the presumed goal of intubating those with a GCS of 8 . Our use of inverse probability weighting was intended to help mitigate the risk of confounding. This statistical method may be a helpful tool in answering a question that is inherently difficult to study. Beyond this, our use of a large national dataset allowed the sample size and power necessary to detect this result and generate a statistically sound study.

We chose to define "intubation" as those who were intubated within an hour of arrival. This may prompt questions about those who potentially undergo slightly delayed intubations in the trauma bay or in the operating room soon after trauma bay evaluation. To determine whether we were missing a significant number of patients intubated outside of the first hour, we re-examined the data for the codes listed in Table 5 in Appendix 1, timestamped for the 2nd and 3rd hours after arrival. Of the 2598 patients who did not meet our initial criteria for ED intubation, 133 (5.1\%) were intubated in the ensuing two hours. This relatively small number of delayed intubations is reassuring.

There are a few limitations to address. The GCS provided in the NTDB is at the time of arrival to the emergency department; we do not know whether this is the best or worst GCS for that patient, nor whether some of these patients may have received sedating medications on route. However, prehospital intubations were excluded and it seems unlikely that many non-intubated patients would have received drugs in the field. Secondly, we are not able to exclude confounding by indication as a possible reason for the association between intubation and mortality we describe. For instance, some physical findings that may be considered indications 
for intubation (e.g., anisocoria, vomiting with concerns for aspiration, evidence of smoke inhalation) are not captured in the data but are associated with mortality. However, as the NTDB does not contain data on indication for intubation, this a known limitation of the dataset. The fact that similar results were shown on an analysis stratified by head injury is encouraging. The $23.3 \%$ missingness rate for intoxication is also limiting, as this might be an additional confounder. Despite this limitation, we are encouraged by the similarity between the stratified and overall results.

We do not intend to suggest that there are no trauma patients with a GCS between 6 and 8 who would benefit from intubation. Furthermore, it should be emphasized that this data only applies to those with a marginal GCS, and not those with a severely depressed mental status. We do, however, believe that this data should prompt trauma providers to question the blanket recommendation that all patients with a GCS of 8 be intubated and use clinical judgement to determine which ones will benefit. Though some patients in this category will surely benefit, we have demonstrated here that for patients who have a GCS of 6-8, after adjustment for severity of injury, those who were observed without intubation suffered no increase in mortality risk and may have in fact been subject to a lower risk. Future directions to further elucidate the appropriate indications for intubation should include dedicated prospective studies that not only question what GCS score is the most appropriate threshold, but also investigate which other, more nuanced factors are appropriate to factor in to the decision of whether or not to intubate.

\section{Conclusion}

Among patients with GCS of 6-8, intubation on arrival was associated with increased mortality, ICU days, and overall length of stay. Future research should be conducted to develop evidence-based guidelines defining the population of trauma patients who may benefit from intubation for decreased level of consciousness.

Author contributions JSH, RPD, EJK, and DNH: conceptualization, data interpretation, and drafting of the manuscript. DS and JBS: critical review and revision of the manuscript.

Funding During portions of this work, DNH was supported by a training grant through the National Heart, Lung, and Blood Institute. (K08HL131995).

\section{Compliance with ethical standards}

Conflict of interest No authors have conflicts to declare.

\section{Appendix 1}

See Table 5 .

Table 5 Procedure codes indicative of endotracheal intubation

\begin{tabular}{lll}
\hline ICD-9 and ICD-10 codes for endotracheal intubation & ICD-10 \\
\hline $\begin{array}{l}\text { ICD-9 } \\
\text { Insertion of endotracheal } \\
\text { tube }\end{array}$ & OBH17EZ & $\begin{array}{c}\text { Insertion of endotracheal airway into trachea, via natural or artificial } \\
\text { opening } \\
\text { Insertion of endotracheal airway into trachea, via natural or artificial } \\
\text { opening endoscopic } \\
\text { 1GZ31CAEP (ICD-10-CA/CCI) } \\
\text { 1GZ31CAND (ICD-10-CA/CCI) } \\
\text { endotracheal intubation, manual hand assisted } \\
\text { Ventilation, respiratory system NEC, invasive per orifice approach by } \\
\text { endotracheal intubation, positive pressure }\end{array}$ \\
\hline
\end{tabular}

ICD-9 International Classification of Diseases, 9th edition, ICD-10 International Classification of Diseases, 10th edition, ICD-10-CA/CCI International Classification of Diseases, 10th edition, as modified for Canadian Use, and the Canadian Classification of Health Interventions; NEC, not elsewhere classified 


\section{References}

1. Committee on Trauma, American College of Surgeons. In: Advanced Trauma Life Support Student Course Manual. Chicago, IL: American Collegeof Surgeons; 2018.

2. Dunham MC, Barraco RD, Clark DE, Daley BJ, Davis FE III, Gibbs MA, et al. Guidelines for emergency tracheal intubation immediately after traumatic injury. J Trauma Acute Care Surg. 2003;55:162-79.

3. Mayglothling J, Duane TM, Gibbs M, McCunn M, Legome E, Eastman AL, et al. Emergency tracheal intubation immediately following traumatic injury: An Eastern Association for the Surgery of Trauma practice management guideline. J Trauma Acute Care Surg. 2012;73:S333-S34040.

4. Sise MJ, Shackford SR, Sise CB, Sack DI, Paci GM, Yale RS, et al. Early intubation in the management of trauma patients: indications and outcomes in 1,000 consecutive patients. J Trauma Acute Care Surg. 2009;66:32-40.

5. Bendinelli $\mathrm{C}, \mathrm{Ku} \mathrm{D}$, King KL, et al. Trauma patients with prehospital Glasgow Coma Scale less than nine: not a homogenous group. Eur J Trauma Emerg S. 2019; . https://doi.org/10.1007/ s00068-019-01139-9.

6. Bendinelli C, Ku D, Nebauer S, King KL, Howard T, Gruen R, et al. A tale of two cities: prehospital intubation with or without paralysing agents for traumatic brain injury. ANZ J Surg. 2018;88:455-9.

7. Committee on Trauma, American College of Surgeons. In: 2016 National Trauma Data Bank (NTDB). Chicago, IL, 2016.

8. Haider AH, Saleem T, Leow JJ, Villegas CV, Kisat M, Schneider EB, et al. Influence of the National Trauma Data Bank on the study of trauma outcomes: is it time to set research best practices to further enhance its impact? J Am Coll Surg. 2012;214:756-68.

9. White IR, Royston P, Wood AM. Multiple imputation using chained equations: issues and guidance for practice. Stat Med. 2011;30:377-99.
10. Rosenbaum PR, Rubin DB. The central role of the propensity score in observational studies for causal effects. Biometrika. 1983;70:41-55.

11. Robins JM, Hernán MÁ, Brumback B. Marginal structural models and causal inference in epidemiology. Epidemiology. 2000;11(5):550-60.

12. D'Agostino RB. Propensity score methods for bias reduction in the comparison of a treatment to a non-randomized control group. Stat Med. 1998;17:2265-81.

13. Holland PW. Statistics and causal inference. J Am Stat Assoc. 1986;81:945-60.

14. Salvino CK, Dries D, Gamelli R, Murphy-Macabobby M, Marshall W. Emergency cricothyroidotomy in trauma victims. J Trauma. 1993;34:503-5.

15. Ligier B, Buchman T, Breslow M, Deutschman C. The role of anesthetic induction agents and neuromuscular blockade in the endotracheal intubation of trauma victims. Surg Gynecol Obstet. 1991;173:477-81.

16. Trupka A, Waydhas C, Nast-Kolb D, Schweiberer L. Early intubation in severely injured patients. Eur J Emerg Med. 1994;1:1-8.

17. Muakkassa FF, Marley RA, Workman MC, Salvator AE. Hospital Outcomes and Disposition of Trauma Patients Who Are Intubated Because of Combativeness. J Trauma Acute Care Surg. 2010;68:1305-9.

18. Duncan R, Thakore S. Decreased Glasgow coma scale score does not mandate endotracheal intubation in the emergency department. J Emerg Med. 2009;37:451-5.

19. Sauaia A, Moore EE, Crebs JL, Maier RV, Hoyt DB, Shackford SR. Evidence level of individual studies: a proposed framework for surgical research. J Trauma Acute Care Surg. 2012;72:1484-90. 